International Journal of Quantum Information

(C) World Scientific Publishing Company

\title{
FEEDBACK IN QUANTUM COMMUNICATION
}

\author{
GARRY BOWEN \\ Centre for Quantum Computation, DAMTP, University of Cambridge, Wilberforce Road \\ Cambridge CB3 OWA, United Kingdom \\ gab30@damtp.cam.ac.uk \\ Received (Day Month Year) \\ Revised (Day Month Year)
}

\begin{abstract}
In quantum communication feedback may be defined in a number of distinct ways. An analysis of the effect feedback has on the rate information may be communicated is given, and a number of results and conjectures are stated.

Keywords: Feedback; quantum channels; channel capacity.
\end{abstract}

\section{Introduction}

Communication is primarily the generation of correlations between two parties. The transmission of information is undertaken to correlate the received message with an possible set of transmitted messages. In the classical theory of information the maximum rate of information transmission through a memoryless channel is given by a measure of the maximum correlation that may be generated through the channel. The correlation measure is the mutual information between the sender's alphabet and the receiver's output alphabet. Utilizing feedback, where the receiver's output state is communicated noiselessly to the sender cannot increase the maximum asymptotic rate of transmission. The capacity, defined as the maximum rate of information transfer per channel use, cannot then be increased by the use of feedback. ${ }^{1}$

Feedback in quantum information theory becomes a more slippery concept, mainly due to the quantum no-cloning theorem. ${ }^{2}$ The output state of the receiver cannot be copied with perfect fidelity, and so the natural generalisation of the classical feedback scenario cannot take place. A more subtle approach is then needed in both the definition and implications of feedback in quantum communication.

\section{Defining Feedback}

The implementation of classical feedback may be done independently of the receiver. The receiver may thus play either an active or passive role in the feedback protocol. In quantum communication the actual role of the receiver can determine the type of feedback utilized. 


\subsection{Active quantum feedback}

The natural extension to the classical feedback protocol is to assume that the receiver may transmit an arbitrary amount of information noiselessly to the sender. The feedback scenario then may be represented by a noiseless channel from receiver to sender. The receiver may process the output of the channel in any way, and include arbitrary addition information to transmit back to the sender. This extension of the concept of feedback does not change the fact that the capacity of the channel cannot be increased by feedback. The idea of a noiseless feedback channel is useful, however, because it may easily be extended to the quantum case. Active quantum feedback can therefore be defined as the use of a noiseless quantum channel from the receiver to sender. Again, the receiver is unrestricted as to the operations they may perform before transmission through the feedback channel.

\subsection{Passive quantum feedback}

Passive quantum feedback is when the sender receivers information about the output state without that information being communicated to the receiver. Any quantum channel may be modelled by a unitary transformation with an environment in a known initial state. From the Kraus representation ${ }^{3}$ of the channel, a measurement of the environment will give a particular output state determined by the outcome of the measurement on the environment. Alternatively, the entire environment state may be accessible by the sender ${ }^{4}$ (labelled coherent feedback by Winter ${ }^{5}$ ).

As shall be demonstrated later, the known upper bounds for active feedback protocols also apply to any passive feedback protocol. Any gains from passive feedback protocols are thus necessarily less than those possible for known optimal active feedback protocols.

\section{Capacities for Quantum Channels}

The Holevo-Schumacher-Westmoreland theorem ${ }^{6,7}$ gives the achievable rates for memoryless quantum channels. This states that the maximum amount of mutual information that may be generated through a quantum channel $\Lambda$ is bounded by the maximum amount of "classical" correlation that may be shared by states through the channel. For $n$ uses of a channel, the upper bound becomes the regularized term,

$$
C=\lim _{n \rightarrow \infty} \max _{\rho_{R Q} \in \mathcal{D}} \frac{1}{n} S\left(R: \Lambda^{\otimes n} Q\right)
$$

where the quantum mutual information is defined by $S(A: B)=S\left(\rho_{A}\right)+S\left(\rho_{B}\right)-$ $S\left(\rho_{A B}\right)$, for $S(\omega)=-\operatorname{Tr} \omega \log \omega$ the von Neumann entropy of the state. The notation $\Lambda^{\otimes n} Q$ represents the effect of $n$ copies of the channel $\Lambda$ acting on the state $\rho_{Q}$. The maximum is taken over all separable quantum states $\mathcal{D}$. Codes exist that can achieve any rate below $C$, with asymptotically vanishing probability of error. 
The entanglement-assisted classical capacity $C_{E}$ is the rate that classical information may be transmitted through a memoryless channel when both the sender and receiver share an unlimited amount of entanglement prior to transmission..$^{9,10}$ The entanglement-assisted capacity is additive, and hence is given by the single shot expression,

$$
C_{E}=\max _{\rho_{R Q}} S(R: \Lambda Q)
$$

where the maximization is over all bipartite quantum states. The entanglementassisted capacity may be strictly larger than the unassisted capacity, an example being for the noiseless channel where $C_{E}=2 C$ from dense coding. ${ }^{8}$

\section{Feedback and Entanglement}

The role entanglement plays in the utilization of a quantum feedback channel is easily seen from the fact that an arbitrary amount of entanglement may be shared between the two parties using the feedback channel. The capacity ${ }^{\mathrm{a}}$ of the channel utilizing quantum feedback must therefore be at least as large as the entanglementassisted capacity $C^{\mathrm{QFB}} \geq C_{E}$.

When the two parties share prior shared entanglement, any classical feedback may be used along with some shared entanglement to generate a noiseless quantum channel via quantum teleportation. Conversely, a noiseless quantum feedback channel may be used to both share entanglement as well as feedback classical information. Therefore the two scenarios are equivalent, and the entanglement-assisted capacity with classical feedback $C_{E}^{\mathrm{FB}}$ is equal to the capacity with quantum feedback $C^{\mathrm{QFB}}$.

It may be shown that any feedback protocol that utilizes a quantum feedback channel has an additive upper bound given by the maximum conditional quantum mutual information $S\left(R: \Lambda Q \mid R^{\prime}\right)=S\left(\rho_{R R^{\prime}}\right)+S\left(\left(\Lambda_{Q} \otimes \mathbb{I}_{R^{\prime}}\right) \rho_{Q R^{\prime}}\right)-S\left(\rho_{R^{\prime}}\right)-$ $S\left(\left(\Lambda_{Q} \otimes \mathbb{I}_{R R^{\prime}}\right) \rho_{Q R R^{\prime}}\right)$, for any state $\rho_{Q R R^{\prime}}$ that is separable between $R$ and $Q R^{\prime}$. By showing that this quantity is necessarily less than the righthand side of (2), the equality $C^{\mathrm{QFB}}=C_{E}^{\mathrm{FB}}=C_{E}$ is obtained. ${ }^{11}$ For a quantum feedback channel the analogy with the classical theory thus only holds in the case of the entanglementassisted capacity.

Examining the case of coherent passive feedback shows that the maximum conditional quantum mutual information is also an upper bound. This may be demonstrated in a similar way to the proof of the case of active feedback, with the feedback operations changed to reflect the use of passive feedback. It is not known whether the upper bound is tight in this case, although it appears unlikely to be so.

a The corresponding entanglement-assisted capacities for quantum information are related to the classical capacities by a factor of one half. This is achieved by utilizing quantum teleportation and quantum dense coding. 


\section{Classical Feedback and Quantum Channels}

When only classical feedback is allowed between the receiver and sender, no entanglement may be shared through the feedback channel. Indeed, it is known that if the two parties do not generate entanglement through the channel then the use of classical feedback cannot increase the capacity, and $C^{\mathrm{FB}}=C .^{12}$ This criterion obviously includes all product (or non-entangled) coding schemes as well as all entanglement-breaking channels.

Recent work, however, has indicated that the ability to generate a higher rate of entanglement utilizing classical feedback may increase the classical capacity for some classes of quantum channels. ${ }^{13}$ The particular examples for which this has been shown are known as echo-correctable channels. At present, the results for echo-correctable channels rely on the assumption of additivity for the separation between the unassisted and classical feedback assisted capacities.

The relationship between classical feedback and quantum information is somewhat different. The rate that quantum information, in the form of intact quantum states or entanglement, may be transmitted is increased by the use of classical feedback. There may even be a separation between rates that unknown states may be transmitted and the rate that entanglement may be shared through some channels.

\section{Discussion}

As initially stated, communication is primarily the generation of correlations between parties. The use of feedback can increase correlations between the communicating parties. In proving the known cases it is shown that, with sufficient prior shared correlations, feedback cannot increase the correlations between the senders message and the receivers output state. Cases where feedback has a positive contribution to the capacity appear to rely on the increased ability to generate additional correlations, generally assumed to be in the form of shared entangled states.

The implications for classical feedback and classical communication are that the necessary pre-existing correlations are inherent in the code that is utilized in communication. For a quantum feedback channel, entanglement may be shared between the parties, however, if the necessary amount of entanglement is pre-existing the rate cannot be increased further. This relationship provides evidence that the entanglement-assisted capacity is a natural generalization for the quantum information domain.

\section{Acknowledgements}

The author wishes to thank the organisers of FQI04. This work was funded by EPSRC grant numbers GR/S34090/01 and GR/S92816/01.

\section{References}

1. T. M. Cover and J. A. Thomas, Elements of Information Theory (Wiley, New York, 1991). 
2. W. K. Wootters and W. H. Zurek, Nature 299, 802 (1982).

3. K. Kraus, States, Effects and Operations (Springer-Verlag, Berlin, 1983).

4. A. Harrow, Phys. Rev. Lett. 92, 097902 (2004).

5. A. Winter, "Identification via Quantum Channels in the Presence of Prior Correlation and Feedback", quant-ph/0403203.

6. A. S. Holevo, IEEE Trans. Inform. Theory 44, 269 (1998).

7. B. Schumacher and M. D. Westmoreland, Phys. Rev. A 56, 131 (1997).

8. C. H. Bennett and S. J. Wiesner, Phys. Rev. Lett. 69, 2881 (1992).

9. C. H. Bennett, P. W. Shor, J. A. Smolin and A. V. Thapliyal, Phys. Rev. Lett. 83, 3081 (1999).

10. C. H. Bennett, P. W. Shor, J. A. Smolin and A. V. Thapliyal, IEEE Trans. Inform. Theory 48, 2637 (2002).

11. G. Bowen, IEEE Trans. Inform. Theory 50, 2429 (2004).

12. G. Bowen and R. Nagarajan, "On Feedback and the Classical Capacity of a Noisy Quantum Channel", quant-ph/0305176.

13. C. Bennett, I. Devetak, P. Shor, and J. Smolin, "Inequalities and separations among assisted capacities of quantum channels", quant-ph/0406086. 